\title{
ESTRATEGIAS ADOPTADAS EN LA HISTORIA EMPRESARIAL DE DOS ORGANIZACIONES TOLIMENSES ${ }^{1}$
}

\section{STRATEGIES TAKEN IN THE ENTERPRISE HISTORY OF TWO TOLIMA ORGANIZATIONS}

\author{
Milena Johanna Cujiño Ibarra ${ }^{2}$, Mario Enrique Uribe Macías ${ }^{3}$ \\ Universidad del Tolima
}

RECIBIDO: Agosto 18 de 2015

ACEPTADO: Noviembre 11 de 2015

DOI

\begin{abstract}
RESUMEN
El artículo se enmarcó en los campos de estudio: historia empresarial y estrategia. Su objetivo es analizar las principales estrategias que las empresas Velotax S.A. y Mercacentro adoptaron a lo largo de su historia y las incidencias que estas tuvieron. La investigación fue descriptiva mediante estudio de casos, método deductivo y aplicación de entrevistas semidirigidas al representante designado por cada gerencia. Las empresas fueron seleccionadas mediante consenso de panel. Las principales decisiones estratégicas de Velotax S.A. fueron: transformación en cooperativa, inversión de dos mil millones en vehículos, creación de tres sociedades anónimas para apalancar la empresa, remoción del señor Contreras del cargo de gerente y desarrollo de un proceso de preparación para la transición directiva. En Mercacentro: crecimiento y expansión como cadena regional de supermercados, creación de imagen y marca asociadas al concepto de región, creación de marca propia, incorporación de la familia en la administración de la empresa y diseño del protocolo de familia. Ambas empresas han estado sometidas en gran medida a la influencia del empresario (gerente), de quien se ha desprendido la formulación de la estrategia, la cual ha dependido de la época de creación y desarrollo, así como del ambiente competitivo en la industria dentro de la cual se desenvuelve cada empresa.
\end{abstract}

Palabras clave: Estrategia, Historia empresarial, Organizaciones tolimenses.

\begin{abstract}
The paper, Research kind, belonged to enterprises' history and strategy study fields, its objective: analyze the main strategies the enterprises Velotax S.A. and Mercacentro adopted along their history and the incidences those had. Research was descriptive, by study of cases, deductive method, using semi-directed interviews to designated representative by every manager; the enterprises were selected by panel consensus. Some of main Velotax S.A. strategic decisions: its transformation into cooperative; investment of \$2.000 million in vehicles; creation of three shareholder corporations in order to lever the enterprise; dismissal of post of manager Mr. Pedro Pablo Contreras; and develop of a preparation for managerial transition process in the enterprise. With regard to Mercacentro: growth and expansion, becoming a regional supermarket chain; creation of an image and brand associated to region concept; creation of its own-brand; family incorporation in the enterprise management; and the design of a family protocol. Both enterprises have been greatly subdued to entrepreneur (manager) influence, whose has made decision about strategy formulation; these strategies have depended of the creation and development epoch, as well as of competitive environment in the industry every enterprise belong.
\end{abstract}

Keywords: Enterprises' history, Strategy, Organizations from Tolima.

Este artículo se puede referenciar

Cujiño, M. \& Uribe, M. (2016). Estrategias adoptadas en la historia empresarial de dos organizaciones tolimenses. En Desarrollo Gerencial Revista de la Facultad de Ciencias Económicas Administrativas y Contables de la Universidad Simón BolivarColombia, 8(1), 109-122.

1 El artículo es resultado del proyecto de investigación "Historia empresarial de dos organizaciones tolimenses, éxitos y fracasos de su estrategia: un paso en la reflexión", financiado por el Comité de Investigaciones de la Universidad del Tolima.

2 Profesora catedrática Universidad del Tolima. Magister en administración, especialista en gerencia de proyectos, administradora financiera. Correo electrónico: milenitajci@gmail.com.

3 Profesor titular Universidad del Tolima. Estudiante de Doctorado en Gerencia de proyectos, magister en administración, especialista en evaluación social de proyectos, especialista en administración financiera, administrador de empresas. Correo electrónico: meuribem@gmail.com. 


\section{Introducción}

Los antecedentes del departamento datan desde su constitución como Estado Soberano del Tolima el 12 de abril de 1861. Después fue designado como departamento en la Constitución de 1886 y, posteriormente, creado jurídicamente mediante la Ley 01 de 1908. Es así que durante este largo período histórico se han vivido transformaciones económicas, políticas, sociales, culturales, legales, tecnológicas, geográficas y competitivas. Todos estos cambios tienen sentido si se interpretan a la luz de las condiciones propias de la época. En ese orden de ideas, es de resaltar que el desarrollo de un país y de una región está marcado por su historia y en gran parte por su historia empresarial, la cual debe convertirse en una fuente para la comprensión de la región y, a su vez, en una base para la construcción del futuro de la misma.

La historia empresarial se define como "aquella disciplina que explora el pasado del actuar empresarial, es decir, estudia la evolución de las empresas y empresarios" (Dávila, 1997, p.10); de esta manera, son "dos los actores esenciales sobre los cuales recae la historia empresarial [...]: el empresario (como actor económico) y la empresa (tanto el resultado del proceder o actuar empresarial, como su rol económico)" (Betancourt, 2003, p.200). El objetivo de este artículo es identificar las estrategias adoptadas y sus efectos posteriores a lo largo de la historia de dos organizaciones, razón por la cual es importante coincidir con Gil e Ibarra (2014, p.124) y Porter (1998) en cuanto a que la estrategia es "una combinación de los fines (metas) por los cuales se está esforzando la empresa y los medios (políticas) con los cuales está buscando llegar ellos"

No existe evidencia, en la literatura de estudios, de que se hayan ocupado de analizar los éxitos o fracasos de la estrategia de organizaciones en el departamento del Tolima desde la óptica de la historia empresarial. En este sentido, y aun cuando el Tolima a lo largo de los años no ha tenido una posición estratégica en materia de desarrollo económico, contribución al PIB nacional, a las exportaciones, al crecimiento, entre otros; es de vital importancia reconocer la existencia de un conjunto de organizaciones que a nivel empresarial han logrado construir historia en el departamento y, a su vez, se han convertido en fuentes centrales de desarrollo, generación de empleo, contribución fiscal y experiencia empresarial.

Por lo expuesto anteriormente, el problema central que se abordó en la investigación se puede definir como: "las estrategias adoptadas por dos organizaciones tolimenses, que han marcado sus éxitos y sus fracasos a lo largo de su historia". En consecuencia, desde el problema se busca correlacionar la historia alrededor de la estrategia empresarial desde una visión sistémica que, más allá de su propia documentación, esté orientada a establecer sus interrelaciones con las diferentes estrategias implementadas. 
El objetivo general de la investigación se centró en efectuar un análisis basado en la documentación de la historia de dos empresas tolimenses representativas, a partir de la identificación de sus estrategias exitosas y no exitosas. Para tal fin se definieron objetivos específicos relacionados con la elaboración de los referentes teóricos aplicables, la reseña de las empresas, la descripción de las estrategias utilizadas por ellas y el análisis de los rasgos comunes y diferencias fundamentales entre las dos empresas.

Por lo anterior, este artículo presenta las principales estrategias adoptadas por las dos organizaciones estudiadas a lo largo de su devenir. La investigación inicia con la obtención de los principales hitos de la historia de los empresarios y de las empresas para identificar, posteriormente, las decisiones estratégicas tomadas y sus consecuencias asociadas, aspectos que se especifican en el presente artículo.

A manera de limitación es importante aclarar que la investigación no pretendía generalizar los hallazgos, pues se trata del caso específico de dos empresas. Su alcance está definido por la información obtenida que se constituye en el marco de referencia para hacer comparaciones y aplicaciones particulares con respecto a los temas de estrategia e historia empresarial, de acuerdo con las condiciones propias de cada organización estudiada.

\section{Método}

La metodología utilizada en el proyecto de investigación es desagregada a continuación en los ítems diseño, participantes, instrumentos y procedimientos:

\section{Diseño}

Con base en un enfoque cualitativo de investigación, que "se basa en métodos de recolección de datos no estandarizados ni predeterminados completamente" (Hernández, Fernández y Baptista, 2014, p.8) y “puede ofrecer una visión más holística y más global de la realidad social” (Deslauries, 2004, p.22), se realizó una investigación de tipo descriptiva en la medida en que, según Hernández et al. (2014), esta busca “especificar las propiedades, las características y los perfiles importantes de personas, grupos, comunidades o cualquier otro fenómeno que se someta a un análisis" (p.92), lo que significa, en términos de Rubio (2015), que el problema se encuentra debidamente entendido y estructurado. Adicionalmente, es un estudio de casos pues de acuerdo con Bernal (2010) su propósito fue realizar el análisis específico de una unidad (empresa, área, actividad, etc.): a efectos de esta investigación se realizó el análisis de las estrategias tomadas a lo largo de su historia empresarial en cada una de las dos organizaciones seleccionadas. Al respecto, Hernández et al. (2014) afirman que el estudio de caso puede utilizar procesos de investigación cuantitativa, cualitativa o mixta para analizar de manera profunda una unidad de estudio y responder al planteamiento de un problema. 
El método de investigación fue deductivo debido a que a partir del referente teórico se analizó la situación específica de cada una de las dos organizaciones estudiadas, con el fin de volver explícitas sus verdades particulares en el marco de las verdades universales contenidas en la teoría. Este método, según Bernal (2010), "consiste en tomar conclusiones generales para obtener explicaciones particulares [...]; inicia con el análisis de los postulados [...] de aplicación universal y de comprobada validez, para aplicarlos a soluciones o hechos particulares" (p.59).

\section{Participantes.}

Para Hernández et al. (2014), la población es el "conjunto de todos los casos que concuerdan con determinadas especificaciones". En este artículo, por tratarse de un análisis de casos, la población corresponde a las dos organizaciones objeto de estudio, las cuales fueron seleccionadas utilizando la técnica de consenso de panel que consiste en reunir a un grupo de expertos en calidad de panel, a quienes se les somete a una serie de preguntas con participación abierta, es decir, sin secreto de sus identidades. Se basa en el supuesto de que, a diferencia de una sola persona, varios expertos serán capaces de generar mejores conclusiones sobre un asunto (Sapag, Sapag y Sapag, 2014).

En cada una de las empresas el gerente designó a un directivo que, por su experiencia y conocimiento, fue el encargado de proporcionar la información requerida para el proyecto de investigación.

\section{Instrumentos}

La primera técnica utilizada fue el Consenso de panel que tuvo como fin seleccionar las dos empresas que serían objeto de estudio de acuerdo con el criterio de los expertos invitados: el gerente regional de la Asociación Nacional de Industriales ANDI, el director de la Federación Nacional de Comerciantes FENALCO, el director regional del Servicio Nacional de Aprendizaje SENA, el presidente ejecutivo (E) de la Cámara de Comercio de Ibagué y el Gerente de Multicentro y Presidente de Actuar Tolima. El instrumento utilizado fue la Guía de trabajo - Panel de expertos (apéndice A).

Posteriormente, para el desarrollo de la investigación y con el fin de recolectar los datos, se acudió a la entrevista semidirigida con el representante de cada empresa, en varias sesiones de trabajo. Esta se basa "en una guía de asuntos o preguntas y el entrevistador tiene la libertad de introducir preguntas adicionales para precisar conceptos u obtener mayor información" (Hernández et al., 2014, p.403). Por medio de este instrumento se indagó sobre el empresario, la empresa, las estrategias adoptadas y los efectos que estas tuvieron en la empresa.

\section{Procedimientos}

De acuerdo con los instrumentos aplicados, en principio se solicitó, en cita personal con cada gerente, 
su autorización para adelantar el estudio; en ambos casos la respuesta fue positiva. Los resultados obtenidos de la aplicación de las entrevistas a sus representantes fueron clasificados de acuerdo con los aspectos mencionados en el ítem anterior: empresario, empresa, estrategias y efectos de las estrategias.

Se desarrollaron cuatro etapas: la primera, dedicada a la construcción del marco teórico y conceptual; la segunda, relacionada con el diseño de los instrumentos y su aplicación, en el primer caso para definir las empresas y en el segundo para obtener la información primaria, la cual fue complementada con información secundaria relacionada con cada una de las empresas investigadas; la tercera etapa correspondió a la organización de la información obtenida por parte de cada empresa; la cuarta se centró en el análisis de las estrategias adoptadas y sus efectos en la historia empresarial de las dos organizaciones.

\section{Resultados}

Como se mencionó en el apartado del método, la investigación es de tipo descriptivo, lo cual concuerda con el análisis de los resultados que se presentan a continuación, producto de una investigación cualitativa basada en el método de caso.

\section{Selección de las empresas}

De la aplicación de la técnica de consenso de panel, con la participación de expertos en el conocimiento empresarial de la región, resultaron las dos empresas que se estudiaron en la investigación. De manera previa, a cada experto se le solicitó llevar dos recomendaciones con base en la guía de trabajo (Apéndice A), centrados principalmente en "variables como las siguientes: origen tolimense de la empresa, antigüedad de la empresa en el mercado, presencia regional, recordación de marca o nombre, volumen de operaciones" (Cujiño y Uribe, 2011, p.9)", obteniendo un total de trece empresas sugeridas. En la reunión se escucharon los argumentos de cada uno de los expertos con respecto a su recomendación y, posteriormente, se le solicitó a cada uno que colocara en orden de importancia a dichas empresas y asignara trece puntos a la primera en su lista y así sucesivamente hasta asignar un punto a la última. Los resultados del ejercicio se observan en la Tabla 1. 
Tabla 1. Resultados del consenso de panel con expertos

\begin{tabular}{|c|c|c|c|c|c|c|c|c|c|}
\hline \multirow[b]{2}{*}{ No. } & \multirow[b]{2}{*}{ Empresa } & \multicolumn{6}{|c|}{ PRIORIZACIÓN DE LOS EXPERTOS } & \multirow[b]{2}{*}{ Total } & \multirow[b]{2}{*}{ Clasificación } \\
\hline & & $\begin{array}{c}\text { Experto } \\
1 \\
\end{array}$ & $\begin{array}{c}\text { Experto } \\
2 \\
\end{array}$ & $\begin{array}{c}\text { Experto } \\
\mathbf{3} \\
\end{array}$ & \begin{tabular}{|c|} 
Experto \\
4 \\
\end{tabular} & \begin{tabular}{|c|} 
Experto \\
5 \\
\end{tabular} & $\begin{array}{c}\text { Experto } \\
6 \\
\end{array}$ & & \\
\hline & & & & & & & & & \\
\hline 1 & $\begin{array}{l}\text { C.I. Lozano y } \\
\text { Maldonado Ltda. }\end{array}$ & 4 & 12 & 9 & 12 & 1 & 11 & 49 & 3 \\
\hline 2 & Cooperamos & 10 & 4 & 13 & 9 & 1 & 6 & 43 & 6 \\
\hline 3 & $\begin{array}{l}\text { Corporación } \\
\text { Forestal del Tolima }\end{array}$ & 8 & 5 & 1 & 3 & 1 & 1 & 19 & 13 \\
\hline 4 & Fibratolima & 9 & 2 & 11 & 4 & 1 & 2 & 29 & 11 \\
\hline 5 & $\begin{array}{l}\text { Granja Buenos } \\
\text { Aires }\end{array}$ & 13 & 7 & 3 & 8 & 1 & 12 & 44 & 5 \\
\hline 6 & Industrias Aliadas & 2 & 9 & 5 & 5 & 13 & 4 & 38 & 8 \\
\hline 7 & $\begin{array}{l}\text { Manufacturas } \\
\text { Carolina }\end{array}$ & 3 & 10 & 6 & 2 & 11 & 5 & 37 & 9 \\
\hline 8 & Mercacentro & 5 & 13 & 12 & 13 & 1 & 10 & 54 & 2 \\
\hline 9 & $\begin{array}{l}\text { Molino Unión de } \\
\text { Arroceros }\end{array}$ & 7 & 8 & 4 & 11 & 12 & 7 & 49 & 3 \\
\hline 10 & $\begin{array}{l}\text { Procesadora de } \\
\text { Pollos Garzón } \\
\text { Ltda. }\end{array}$ & 1 & 11 & 7 & 7 & 9 & 8 & 43 & 6 \\
\hline 11 & Sida Ltda. & 6 & 1 & 8 & 1 & 1 & 9 & 26 & 12 \\
\hline 12 & Sorroza y Suárez & 12 & 6 & 2 & 6 & 1 & 3 & 30 & 10 \\
\hline 13 & Velotax & 11 & 3 & 10 & 10 & 10 & 13 & 57 & 1 \\
\hline & $\begin{array}{l}\text { No fue priorizada } \\
\text { por el experto; se } \\
\text { asume } 1 \text { punto }\end{array}$ & & & & & & & & \\
\hline
\end{tabular}

Fuente. Cujiño I., M. y Uribe M, M. (2014, p. 60)

De acuerdo con lo anterior, las dos empresas seleccionadas fueron Velotax S.A. y Mercacentro. La primera es una empresa de transporte de pasajeros y de carga; la segunda, una cadena de supermercados.

\section{Historia empresarial de cada empresa.}

\section{$\checkmark$ Velotax S.A.}

Don Pedro Pablo Contreras es el fundador de Velotax y es considerado, por quienes lo conocen y por quienes han trabajado con él, como un patriarca ${ }^{1}$, que a partir de una oportunidad creó una empresa que ha

1 Persona que por su edad y sabiduría ejerce autoridad moral en una familia o colectividad 
sobrevivido, crecido y se ha posicionado en su sector y ha aportado al desarrollo personal, económico y social de muchas personas que han estado alrededor de ella durante estos sesenta años.

Ilustración 1. Logotipo de Velotax S.A.

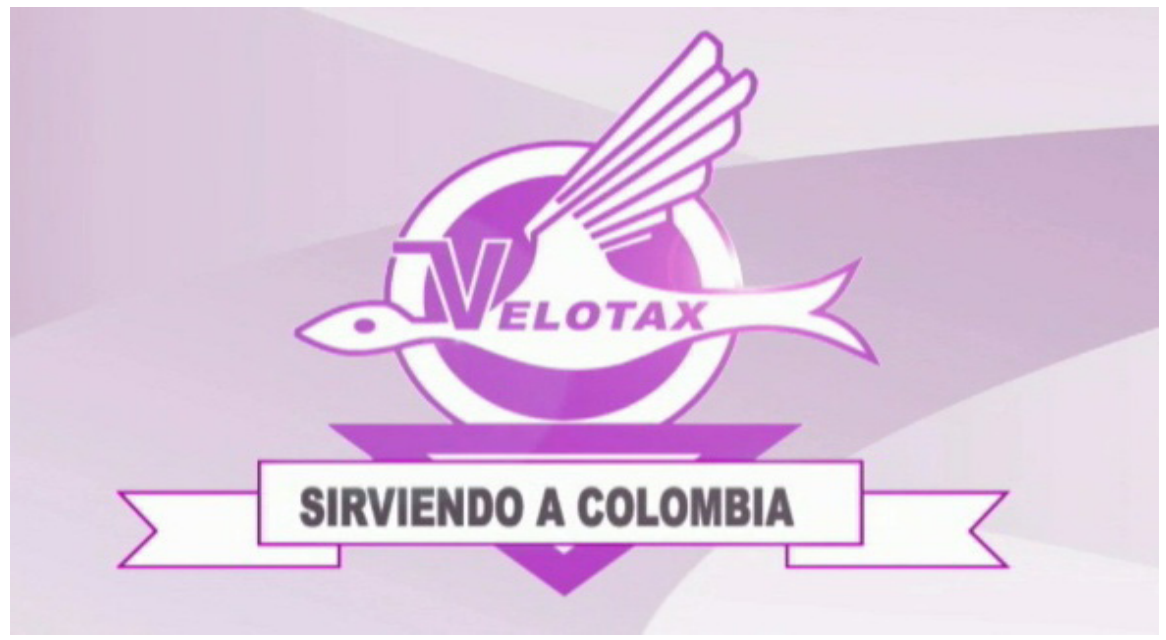

Fuente. Subgerencia de Velotax

Don Pedro Pablo nació en 1920 en el seno de una familia campesina con propiedades en Calarcá (Quindío) y una buena posición económica, lo que le permitió adquirir su propio vehículo (extraño para la época), que lo llevaría más adelante, en 1953, a fundar Velotax.

Esta empresa se creó con la participación de sus amigos, al inicio como una empresa de taxis de servicio urbano que después pasó a encargarse del transporte intermunicipal. Más tarde, realiza la importación de un lote de vehículos que son incorporados a la flota de la empresa. Después de la época de la violencia, don Pedro Pablo transforma la empresa en una cooperativa, de nuevo con la participación de sus amigos cercanos, algunos de los cuales recibieron un vehículo para la operación del negocio.

En 2005, la empresa estuvo al borde de la quiebra de la cual se salvó al refinanciar un leasing que tenía alto costo financiero y que era la causa de la crisis por la que pasaba la cooperativa. El empresario, don Pedro Pablo, crea tres sociedades anónimas con el fin de prestar y vender servicios a Velotax y apalancarla financieramente.

En 2011 se empieza a gestar un movimiento al interior de la Asamblea General encaminado a retirar de la gerencia a don Pedro Pablo (quien siempre la había ejercido), que termina en su separación del cargo por decisión del Consejo de administración. Sin embargo, ante las instancias judiciales, don Pedro Pablo logra ser reintegrado. 
Velotax tiene, con corte al año 2014, 300 asociados, 1.000 vehículos, 150 oficinas en el país, 1.500 empleados, ventas de $\$ 100.000$ millones anuales, transporta 8.000 pasajeros diarios, tiene rutas nacionales en Ibagué, Bogotá, Pereira, Cali, Armenia y Villavicencio, y locales en Líbano, San Antonio, Purificación, Prado, San Luis, Roncesvalles y La Dorada. El transporte de carga se realiza en quince departamentos y se hacen 15.000 despachos diarios de carga.

La empresa está desarrollando un modelo de transición, formando los cuadros directivos que deberán hacer el relevo, solucionando los problemas existentes y planeando la reforma de estatutos para poner la cooperativa a tono con los nuevos retos modernos.

\section{$\checkmark$ Mercacentro}

El fundador de Mercacentro es don Carlos Alvarado, un quindiano que inició sus actividades empresariales en Planadas (Tolima) con la apertura de una tienda. Posterior a la época de la violencia, se trasladó a Ibagué y colocó un granero que después se constituiría en el primer supermercado de la cadena, en septiembre de 1991.

\section{Ilustración 2. Logotipo de Mercacentro}

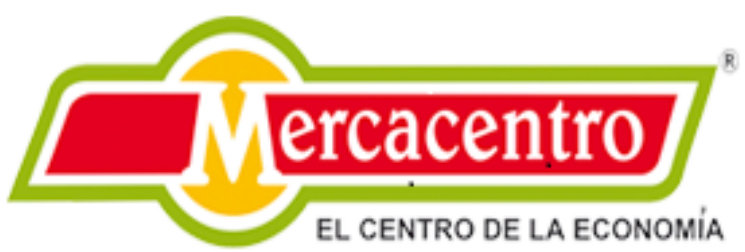

Fuente. Página web

Después, entre 1995 y 1999, adquiere un par de supermercados que estaban en declive y que planeaban cerrarse. En 2001 abre sus puertas Mercacentro 4, formato nuevo para la cadena, con el fin de competir con los hipermercados existentes y los que se avizoraban llegar a la ciudad de Ibagué.

A la par de este nuevo punto de venta, Mercacentro inicia su campaña de regionalismo, que continúa en el presente y genera un alto nivel de fidelidad entre los clientes. La cadena ha seguido creciendo de manera sostenida, abriendo más puntos de venta estratégicamente situados, hasta completar ocho almacenes (en 2013), uno de ellos en el cercano municipio de Espinal.

Como acciones de competencia frente a los grandes formatos, crea su marca propia, genera programas de fidelización (tarjeta para acumular compras y tarjeta de crédito en alianza con una entidad financiera) y promueve la producción local (como parte de su campaña de región). 
Esta es una empresa de familia que se ha venido fortaleciendo administrativamente: en 2005 acuerdan y expiden el protocolo de familia, mediante el cual se regla la participación de los diferentes miembros en la dirección y gestión de la empresa. En los últimos tiempos, en los años 2013 y 2014, han desarrollado un proceso de redireccionamiento estratégico y se encuentran implementando su sistema de gestión de calidad, a la luz de la norma ISO 9001.

\section{Estrategias adoptadas.}

\section{$\checkmark$ Velotax S.A.}

Su primera gran estrategia fue transformar la empresa en una Cooperativa. Esta estrategia se adoptó, además de la orientación al cumplimiento de la ley, con el fin de eliminar la carga impositiva para la empresa. De acuerdo con la legislación colombiana (Ley 863 de 2003), las cooperativas no están obligadas a pagar al Estado el impuesto de renta y complementarios, lo cual genera un alivio en los costos totales finales de la organización.

Este alivio, inmediato y positivo, fue efecto directo de la decisión estratégica. Sin embargo, una cooperativa, dada su esencia, no es de nadie: es de todos; su modelo indica que cada asociado, independientemente del valor de su aporte, tiene un voto y por lo tanto decide en igualdad de condiciones. Surge, entonces, la figura del empresario don Pedro Pablo, quien, no obstante el modelo cooperativo, continuó dirigiendo la organización a sus anchas, si se permite el término, pues, no obstante la forma jurídica, todos (asociados, empleados, clientes y ciudadanía en general) continuaron considerando a Velotax como la empresa de don Pedro Pablo.

En ese entonces, se perdió de vista que tal situación no sería eterna, pues recientemente (en 2013) generó otro impacto que, según el punto de vista, puede ser negativo o positivo. Debido a la decisión estratégica (más de don Pedro Pablo que de la Cooperativa) de crear tres empresas para venderle servicios y productos a Velotax, algunos asociados (dueños de la empresa según el modelo cooperativo) decidieron emprender acciones para separar de la gerencia de la empresa a don Pedro Pablo. Y a fe que lo lograron, pero por vicios de forma este último logró permanecer en su cargo. Se podría percibir como una rencilla interna por el poder, pero en realidad ha generado un alto impacto en la imagen de la empresa, pues estas situaciones trascienden a la opinión pública y lesionan internamente la dinámica de la organización y su clima interno.

La segunda gran decisión estratégica que merece resaltarse corresponde a la decisión de crear tres empresas en cabeza de don Pedro Pablo. Velotax, por su situación económica (vivió una crisis en 2005 - 2006) y por su forma jurídica, empezó a tener inconvenientes para apalancarse financieramente, lo cual ahogaba la 
operación diaria. Con el fin de ayudar en esta situación, su gerente crea tres empresas privadas, regidas por el Código de Comercio, que buscan oxigenar a Velotax pues son sujetas de crédito y cuentan con adecuados niveles de liquidez. Estas empresas surten de repuestos e insumos a Velotax y de locales para su operación, entre otros, lo cual ayuda a aligerar algunos procesos de la organización.

Pero también generan cuestionamientos y malquerencias de algunos asociados, con las consecuencias descritas anteriormente. No obstante el impacto negativo, hay una consecuencia positiva importante que surge de la decisión: por primera vez en su historia, Velotax, de una manera intencionada y planificada, empieza a desarrollar un proceso para el relevo generacional directivo que busca preparar de manera adecuada a la persona que deberá suceder a don Pedro Pablo, cuando sea el momento.

\section{$\checkmark$ Mercacentro}

La primera gran decisión estratégica es la de expansión y crecimiento. En un período de tiempo relativamente corto (1991 - 2013), los supermercados Mercacentro pasaron de tener un solo establecimiento a poseer ocho. En la mayoría de los casos se logró al aprovechar, de manera inteligente, oportunidades que don Carlos Alvarado detectó en su entorno. Puede afirmarse que, salvo la construcción de Mercacentro 4 (para competir con los hipermercados de la ciudad), el crecimiento ha estado basado en la estrategia de adquisición. Sus resultados saltan a la vista, pues en términos de cobertura, participación en el mercado, posicionamiento e imagen; la empresa ha mejorado considerablemente. Además, cada supermercado cuenta con sus propios segmentos de mercado y clientes que les son fieles.

Una gran apuesta que Mercacentro hizo y sigue cultivando de manera estratégica es el concepto de región: "Mercacentro es de todos... es de aquí". Esta decisión la ha llevado a tener el aprecio de los consumidores tolimenses y a generar oportunidades para que los pequeños empresarios de la región tengan una posibilidad interesante de mercado que no les sería posible en otra gran superficie de la ciudad. En Mercacentro se encuentra oferta tolimense de variadas líneas y variados productos. Además, es patrocinador del equipo de fútbol de la región, el Club Deportes Tolima.

Por efectos de la competencia del sector y de acuerdo con las tendencias existentes, Mercacentro tomó otra decisión estratégica importante: crear su marca propia. A la par de las grandes cadenas, hoy en día Mercacentro es una marca que los clientes reconocen y aprecian, pues sus productos se venden a un precio inferior a los demás del mercado, con buena calidad y con impactos sociales positivos dadas las familias que reciben su sustento de parte de los productores que trabajan para la marca.

En la marca propia pueden encontrarse productos como arroz, pastas alimenticias, café, chocolate, ce- 
reales, avena, condimentos, gelatina, granola, maíz pira, panes y galletas frescos, pan tajado, pastelería, postres, vinos, elementos de aseo en general, implementos para mascotas, papel higiénico, harinas para preparar buñuelos y natilla, leche en polvo, entre otros.

Como puede observarse, las estrategias han sido particulares y variadas, con especificidades derivadas del sector en el que compite cada empresa, así como de las exigencias del entorno en cada época.

\section{Discusión}

Dávila (1997) plantea que la historia empresarial explora el pasado del actuar empresarial, es decir, estudia la evolución de las empresas y los empresarios. Lo anterior se evidencia en el análisis realizado, ya que estudiar a Velotax S.A. es centrarse en la historia de don Pedro Pablo Contreras e investigar a Mercacentro es analizar la evolución como empresario de don Carlos José Alvarado Parra. El empresario y la empresa están indefectiblemente unidos en su historia empresarial y así es percibida en la región la historia de Velotax S.A., que es la historia de don Pedro Pablo, y la historia de Mercacentro, que es la historia de don Carlos.

Vale la pena considerar al empresario desde diferentes ópticas y autores: Schumpeter (1968), citado por Torres (2013, p.1), "caracteriza al empresario como un innovador”; Mintzberg (1993), citado por Cujiño y Uribe (2014, p.28), "agrupa los roles de los directivos en relaciones interpersonales, transferencia de información y toma de decisiones"; McClelland (1968), citado por Cujiño y Uribe (2014, p.28), "señala que el empresario es alguien que ejerce control sobre los medios de producción y produce más de lo que consume con el fin de intercambiarlo y lograr un beneficio propio". La historia del empresario se enmarca dentro de la historia empresarial que según Gideut (2009) "es la certidumbre de que el pasado inevitablemente influye en las decisiones del presente, tanto en los negocios como en otras esferas de la actividad individual, bien en un sentido positivo, bien como reacción contra la experiencia del pasado" (p.5).

La historia empresarial de Velotax S.A. es más extensa y complicada que la de Mercacentro. Mientras que en la primera se han presentado inconvenientes de tipo político-administrativo, financiero y de cohesión social; en la segunda las cosas han marchado en una curva ascendente de desarrollo que es percibida por la comunidad en general.

En cuanto a las empresas, vale resaltar que Velotax, que es una cooperativa, se siguió percibiendo, desde adentro y desde afuera, como la empresa de don Pedro Pablo, dada su altísima capacidad de manejo en las relaciones con todas las partes interesadas. 
Mercacentro es la típica empresa de familia que se da en la región y en el país, la cual inicia con la figura del emprendedor y en la medida en que evolucionan empresa y familia, sus miembros se van incorporando a la gestión de la empresa hasta copar todos sus cargos directivos.

El desarrollo de la historia empresarial en cualquier región está marcado por las decisiones que las empresas hayan tomado durante su vida, lo que implica que las estrategias conducen a la supervivencia y a la continuidad en el largo plazo. Al respecto, Johnson, Scholes y Whittington (2014) plantean que "las decisiones estratégicas son aquellas relacionadas con, y que afectan a, la dirección a largo plazo de una organización. Las decisiones estratégicas hacen referencia, normalmente, a cómo lograr ciertas ventajas para la organización" (p.4); planteamiento que coincide con Kluyver (2001) en cuanto la estrategia tiene que ver con posicionar a una organización para que alcance una ventaja competitiva sustentable. Serna (2014) afirma que la estrategia es un conjunto de acciones que se realizan para mantener y apoyar el logro de los objetivos organizacionales, y David (2012) complementa en la medida en que las estrategias son los medios mediante los cuales los objetivos de largo plazo serán alcanzados. Zerón, Sánchez y Guadalupe (2015, p.21) afirman que Porter "reconoce el impacto de las decisiones directivas en la estrategia de la empresa" mediante la cual se puede alcanzar una adecuada posición competitiva en el mercado.

Sobre este marco es importante revisar las principales estrategias adoptadas por las dos organizaciones en estudio.

Algunas de las principales decisiones estratégicas de Velotax S.A. son: su transformación desde el punto de vista jurídico en cooperativa; la adquisición, en 2005, de vehículos por valor de \$2.000 millones; la creación de tres sociedades anónimas, en cabeza de su gerente, para apoyar las actividades de la empresa; la remoción del cargo de gerente de don Pedro Pablo Contreras y el desarrollo de un proceso de preparación para la transición directiva en la empresa.

En cuanto a Mercacentro, pueden mencionarse las siguientes decisiones estratégicas: crecimiento y expansión, constituyéndose en una cadena de supermercados de origen regional; la creación de una imagen y marca asociadas al concepto de región; la creación de su marca propia de productos, al igual que las grandes superficies de orden internacional; la incorporación de los miembros de la familia en la administración de los negocios de la empresa y el diseño del protocolo de familia.

En base a lo anteriormente planteado, se concluye que:

- Velotax S.A., que nació en 1953 como una empresa de taxis, hoy en día es uno de los grandes competidores en la industria de transporte de pasajeros y de carga en el centro del país. 
- Mercacentro nació en 1991, pero a la vuelta de veintidós años ya contaba con ocho supermercados, convirtiéndose así en la única cadena de supermercados de origen tolimense.

Cabe afirmar que al analizar las diferentes decisiones estratégicas adoptadas por cada empresa, se evidencia que estas han estado influenciadas por la figura del empresario (don Pedro Pablo Contreras y don Carlos Alvarado, en cada caso), por las circunstancias de tiempo (época) en la cual cada una nació y se desarrolló y por las características de su actividad económica y el ambiente competitivo en el que se desenvuelven.

Con el adelanto de la investigación surgen algunas inquietudes con respecto al desarrollo de futuros estudios que contribuyan a fortalecer el conocimiento del tema y el aporte que este puede proporcionar a los procesos formativos a nivel académico y empresarial. En el ejercicio realizado con los expertos para definir estas dos empresas quedaron en lista otras once organizaciones sobre las cuales podría desarrollarse un estudio similar: C.I. Lozano y Maldonado Ltda. (Marca: Camisas Monarca), Molino Unión de Arroceros, Granja Buenos Aires (liquidada), Cooperamos, Procesadora de Pollos Garzón Ltda. (Marca: Pollosgar), entre otras con menor puntaje y apreciación por parte del equipo de expertos.

De igual manera se podría desarrollar un nuevo ejercicio con la técnica de consenso de panel para actualizar la percepción de los expertos sobre aquellas organizaciones que en la actualidad podrían ser objeto de un proyecto de investigación similar al adelantado.

\section{Referencias}

Bernal, C. (2010). Metodología de la investigación. Administración, economía, humanidades, ciencias sociales. ( $3^{\mathrm{a}}$ ed.). Bogotá: Pearson Educación.

Betancourt, G. (2003). De la historia empresarial a la historia organizacional. Innovar, revista de ciencias administrativas y sociales, 22, 199-210.

Cujiño I., M. y Uribe M., M. (2014). La estrategia en la historia empresarial: análisis de dos casos empresariales. Ibagué: Universidad del Tolima.

Cujiño I., M. y Uribe M., M. (2011). Historia empresarial de dos organizaciones tolimenses, éxitos y fracasos de su estrategia: un paso en la reflexión. Proyecto de investigación. Ibagué: Universidad del Tolima.

David, F. (2012). Conceptos de administración estratégica. (14a ed.). México: Pearson Educación.

Dávila, C. (1997). Historia empresarial de Colombia: estudios, problemas y perspectivas. Monografía No. 20 Serie Historia empresarial. Bogotá: Universidad de los Andes. 
Deslauries, J. (2004). Investigación cualitativa guía práctica. Pereira: Papiro.

Gideut. (2009). Empresas industriales creadas por emprendedores tolimenses durante el período 1980 2000: análisis crítico de los factores que incidieron en su desaparición. Proyecto de investigación. Ibagué: Universidad del Tolima.

Gil, I., e Ibarra, S. (2014). Incidencia del liderazgo en los factores críticos del éxito como estrategia competitiva empresarial. Dimensión empresarial, 12(2), 117-126.

Hernández, R., Fernández, C. y Baptista M. (2014). Metodología de la investigación. (6 ${ }^{\mathrm{a}}$ ed.). México: McGraw-Hill.

Johnson, G., Scholes, K., y Whittington, R. (2014). Dirección estratégica. (7ª ed.). Madrid: Prentice Hall. Kluyver, C. (2001). Pensamiento estratégico. Buenos Aires: Prentice Hall.

Ley 863 de 2003. Modificación del estatuto tributario colombiano. Recuperado de: http://www.alcaldiabogota.gov.co/sisjur/normas/Norma1.jsp?i=11172.

Porter, M. (1998). Estrategia competitiva. México: CECSA.

Rubio, G. (2015). Modelo de gestión integral de operaciones desde la complejidad. Desarrollo Gerencial Revista de la facultad de ciencias económicas, administrativas y contables de la Universidad Simón Bolívar, 7(2), 153-169. http://dx.doi.org/10.17081/dege.7.2.1186.

Sapag, N., Sapag, R., y Sapag, J. (2014). Preparación y evaluación de proyectos. (6 ed.). México: Mc.GrawHill.

Serna, H. (2014). Gerencia estratégica. (11ª.ed.). Bogotá: 3R Editores.

Torres, L. (2013). Marco referencial. Documento sin publicar. Florencia.

Zerón, M., Sánchez, D., y Guadalupe, N. (2015). Estrategias de negocio de las pymes familiares en ciudad Victoria, Tamaulipas-México. Desarrollo Gerencial Revista de la facultad de ciencias económicas, administrativas y contables de la Universidad Simón Bolívar, 7(2), 19-35. http://dx.doi.org/10.17081/ dege.7.2.1178. 